\title{
The Use of Dynamic Message Signs (DMSs) on the Freeways: An Empirical Analysis of DMSs Logs and Survey Data
}

\author{
Boniphace Kutela, Hualiang Teng \\ Department of Civil Engineering, University of Nevada, Las Vegas, USA \\ Email: kutela@unlv.nevada.edu, Hualiang.Teng@unlv.edu
}

How to cite this paper: Kutela, B. and Teng, H.L. (2021) The Use of Dynamic Message Signs (DMSs) on the Freeways: An Empirical Analysis of DMSs Logs and Survey Data. Journal of Transportation Technologies, 11, 90-107.

https://doi.org/10.4236/jtts.2021.111006

Received: November 30, 2020

Accepted: January 25, 2021

Published: January 28, 2021

Copyright (c) 2021 by author(s) and Scientific Research Publishing Inc. This work is licensed under the Creative Commons Attribution International License (CC BY 4.0).

http://creativecommons.org/licenses/by/4.0/

\begin{abstract}
This study evaluates the Dynamic Message Signs (DMSs) use to dissipate incident information on the freeways in Las Vegas, Nevada. It focuses on the DMSs message timing, extent, and content, from the operators' and drivers' perspectives, considering the variability in drivers' freeway experience. Twoweek incidents data with fifty-nine incidents, DMS log data, and responses from a survey questionnaire were used. The descriptive analysis of the incidents revealed that about $54 \%$ of the incidents had their information posted on the DMSs; however, information of only $18.6 \%$ of the incidents was posted on time. The posted information covered the incident type (54.2\%), location (49.2\%), and lane blockage (45.8\%), while the expected delay or the time the incident has lasted are rarely posted. Further, the standard DMSs are the most preferred sources of traffic information on the freeway compared to the travel time only DMSs, and the graphical map boards. The logistic regression applied to the survey responses revealed that regular freeway users are less likely to take an alternative route when they run into congestion, given no other information is available. Conversely, when given accurate information through DMSs, regular freeway users are about 2.9 times more likely to detour. Furthermore, regular freeway users perceive that the DMSs show clear information about the incident location. Upon improving the DMSs usage, 73\% of respondents suggested that the information be provided earlier, and $54 \%$ requested improvements on congestion duration and length information. These findings can be used by the DMSs operators in Nevada and worldwide to improve freeway operations.
\end{abstract}

\section{Keywords}

Dynamic Message Signs, Dynamic Traffic Display, Driver Behaviors, Freeways, Detour 


\section{Introduction}

Dynamic Message Signs (DMSs), which are also known as Changeable Message Signs (CMS), Variable message signs (VMS), or Electronic Message Signs (EMS), are devices installed along the roadside to display messages of special events. DMSs, as one of the important ITS devices, have been used for many years in managing incidents by providing real-time traffic information of road networks to drivers to improve route choice and relieve traffic congestion. They warn of congestion, incidents, roadwork zones, or speed limits on a specific highway segment. Several studies [1] [2] show how to present traffic and incident information on DMSs so that travelers can perceive it. These studies and manuals also provide recommendations on installing DMSs, so travelers receiving the information have sufficient space to make a diversion decision. Displaying incident information on freeways than where incidents occur has been specially recommended.

Numerous studies evaluated the effectiveness of the DMS in conveying congestion information so that drivers can divert to alternative routes to avoid traffic congestion. Another study [3] developed a discrete choice model to estimate the probability of route diversion; their study also evaluated the impact of DMS on travel time and congestion delays. The results indicate that the messages on DMS do have a significant impact on route diversion, no influence on the travel time, but significantly reduce total delay for traffic in congestion. Another study [4] evaluated the effectiveness of DMSs about the driver's satisfaction level and impact on work zones, and the impact on diversion. The results indicated that the work zone speed was reduced significantly, and motorists were very satisfied with the DMSs. A study by Haghani et al. [5] found that traffic speed is not influenced by the DMS message. Such a finding is contrary to the findings by Guattari et al. [6], who concluded that the approach speeds to the DMSs decrease by more than $5 \%$ if the sign is not understood. Another study by Yan $\&$ $\mathrm{Wu}$ [7] utilized a driving simulation experiment to determine the effectiveness of DMSs on driving behavior. Using a set of road network with three levels of DMS locations, this study found that driver characteristics, DMS location, and information format greatly influence driving behaviors. Further, the same study [7] revealed that the graphic DMS format is better than the text-only DMS format. They recommended positioning the DMS between $150 \mathrm{~m}$ to $200 \mathrm{~m}$ upstream of the diverging point to leverage the effects of DMSs on traffic safety and operation. In improving route choice in case of traffic incidents on the freeway a study by Demetsky and Schroeder [8] recommended that travel time or the delays estimates for both the primary and alternate routes be provided on DMSs.

It can be observed that regardless of the type of the data and methodology used, researchers agree that for the DMS to be useful, the following factors are important; 1) the location where they are positioned; 2) the type of information displayed; 3 ) the time interval from the incident occurrence and message display; and 4) the distance from the incident location to the DMSs where messages 
are displayed. However, previous studies did not consider the DMSs' log data and variation of preferences and satisfaction of the incident message dissipation approaches per drivers' freeway experience levels. Therefore, this study incorporates DMSs log data and survey questionnaire data to evaluate the usefulness of DMSs in the Las Vegas freeway systems. The survey focuses on the preferences and satisfaction of several incidents' message dissipation options on the freeways for drivers with varying freeway experience. Further, this study investigates whether incident/traffic information is distributed to the motorists at the right time, at the right location, and with the clear content to enable drivers of different levels of freeway experience to take proper actions. The rest of the paper is organized as follows; the next section presents the study methodology followed by incidents and DMS log data evaluation. The survey design, analysis, results, and discussion are then presented. Lastly, the conclusion and recommendations are presented.

\section{Study Methodology}

To properly evaluate the usefulness of the DMSs on the freeways, the research team selected Las Vegas Metropolitan area as the study location. Figure 1 presents the flow chart of the methodology applied in this study. This location was selected since it has several DMSs across the freeways, as shown in Figure 2

\subsection{Study Area Description}

Las Vegas has a cross-structured freeways system and a grid system of the arterial roadways on which the Dynamic Message Signs are located. The Freeway and Arterial System of Transportation (FAST), monitors a total of 82 Dynamic Message Signs, including 14.6\% (12) display travel time only, and the remaining (70) display both travel time and incident information. Spatially, $40 \%$ of the DMSs are located along I-15, whereby 19 DMSs are in the northbound and 14 are in the southbound direction. I-515 and I-215 have 8 and 7 DMSs, respectively, while the other state routes and major arterials share the remaining 34 DMSs Figure 2. Geographically, $61 \%$ of the DMSs are located at the interchange, $21 \%$ just after the interchange, and $18 \%$ just before the interchange in the same direction of travel. The minimum distance between the DMSs located in the same direction of travel is 0.4 miles, while the maximum distance is 13 miles.

Almost $90 \%$ of major arterials connect to freeways by using interchanges, thus, providing the opportunity for taking an alternative route in case of major incidents. On average, the major arterials in Las Vegas have an average posted speed limit of $45 \mathrm{mph}$ and about 1.5 minutes average cycle length traffic signals positioned at about a mile apart. With the DMSs alerting the drivers of road conditions downstream and the arterials' grid system connecting to the freeway, the opportunity to take an alternative route is clearly presented; however, the road users are yet to make use of the information displayed. To explore the possible reasons, the incident and DMSs log data were analyzed. 


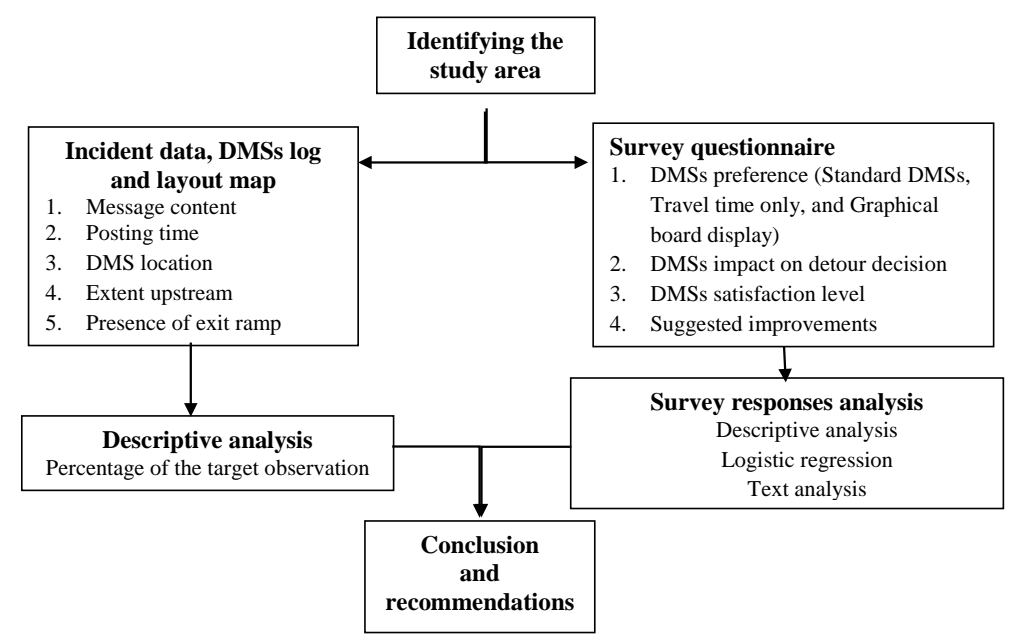

Figure 1. Study methodology flow chart.

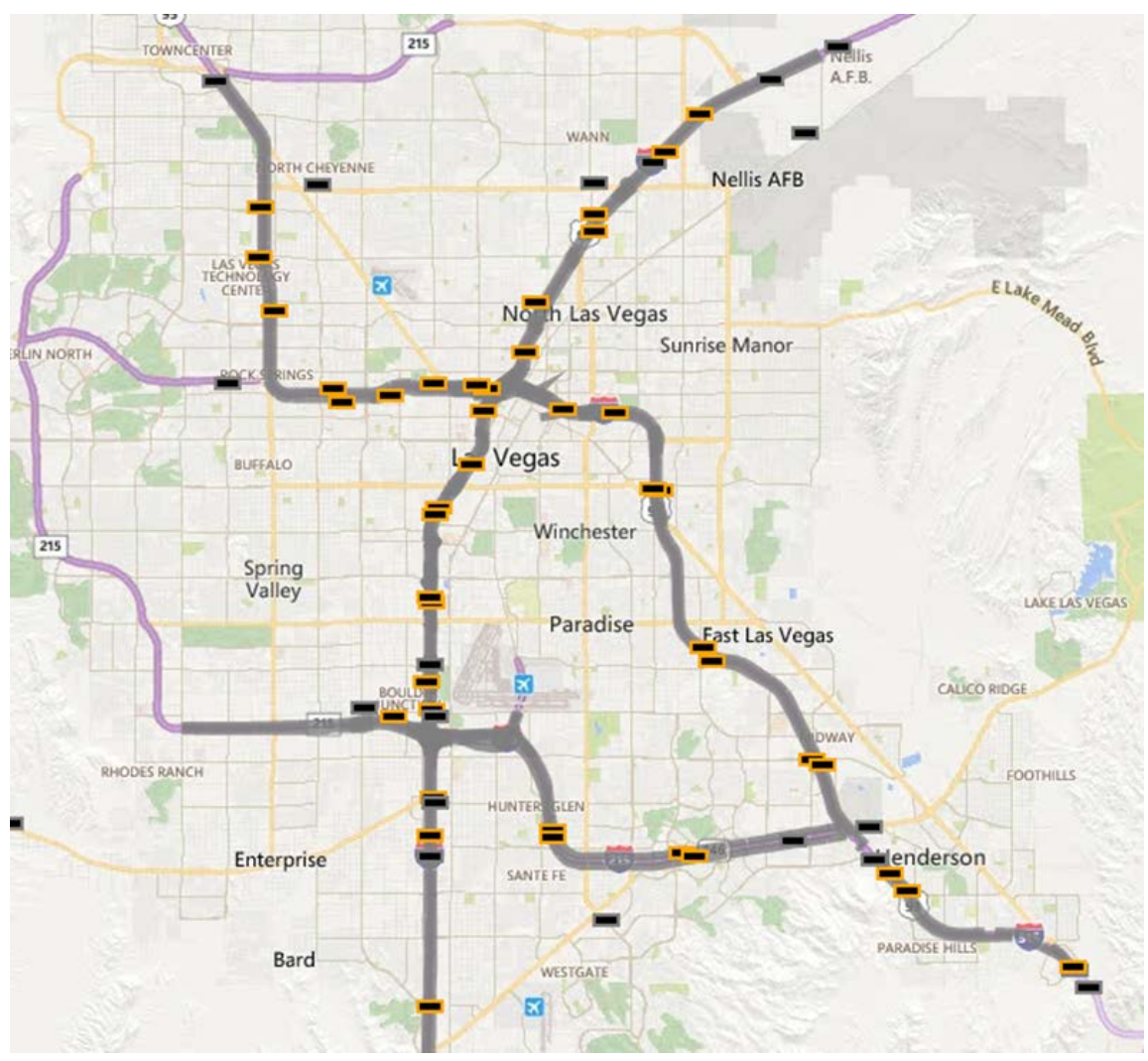

Figure 2. Dynamic message signs locations (Source: [9]).

\subsection{Incident and DMSs Log Data}

The study area in Las Vegas, Nevada, was selected first for which the DMSs and incident datasets for two weeks (December 2-16, 2014) were acquired from the FAST, the agency responsible for overseeing the traffic operations in Las Vegas, Nevada. The incident data contains details such as the incident type, time, location of the incident, and severity in terms of fatality, lane blockage, and duration. The DMSs log data contains the details regarding the message posted on the 
DMS. The DMSs' log details included the time the message was posted, the DMS to which the message was posted, and the exact message posted. These datasets facilitated the determination of the message content and the posting timing (the difference between the incident time and posting time). The DMSs layout map was used together with the DMSs log data to identify the extent upstream the incident location the DMSs were activated.

\subsection{Survey Questionnaire}

Simultaneously, the survey questionnaire was constructed and sent to travelers, most of whom were public workers in the city of Las Vegas, the city of Henderson, and the University of Nevada, Las Vegas community. The Qualtrics platform was used for constructing the survey, and the link to the survey was given to the stated targeted communities. The data collection through qualtrics started on August 11, 2015 and finalized on October $1^{\text {st }}, 2015$. Apart from demographic-related questions, the survey questionnaires included several questions intended to understand drivers' perceptions toward various information dissemination devices. Sample questions include:

1) How frequently do you use highways as opposed to city streets in Las Vegas?

2) When you run into congestion, do you take alternative routes?

3) Do you take an alternative route only when you see slow traffic, regardless of the messages displayed on the dynamic message sign?

4) Would you take an alternative route if you are provided with accurate information about incident/congestion by the dynamic message signs?

5) Please provide any suggestions to make the dynamic message signs more useful to you.

A complete set of survey questions can be found in the following published report [10].

\subsection{Data Analysis Methods}

Three data analysis methods; descriptive analysis, logistic regression, and text analysis, were applied in this study.

\subsubsection{Descriptive Analysis}

The descriptive analysis was applied to both the survey questionnaire and the incident and DMSs log data. The focus for descriptive analysis on DMSs log data was to evaluate three aspects; the message content, timing, and extent of message dissipation upstream. These aspects were then compared to what has been observed through the incident and DMSs data and the ones from the survey questionnaire. For the survey questionnaire data, the descriptive analysis was applied to understand the distribution of the responses.

\subsubsection{Logistic Regression}

To determine the variation in driver's dependency on DMSs for making a decision on taking an alternative route, respondents were asked three questions/ 
scenarios: 1) the general perception towards taking an alternative route when they run into congestion; 2) whether they consider the message posted on the DMSs before deciding on taking an alternative route; and 3) whether they would take an alternative route if the accurate information is provided. In addition, freeway users were asked their perception of the DMSs message clarity regarding location, fatality, blockage, elapsed time, and backup. The respondents were divided into two main groups based on the freeway usage frequency responses; the regular (daily) users and occasional (non-daily) users for further analysis.

For model development, the null hypothesis $\left(\mathrm{H}_{\mathrm{o}}\right)$ is that there is no statistically significant difference among regular and occasional users on the three scenarios stated above. The alternative hypothesis ( $\mathrm{H} 1)$ is there is a statistically significant difference. The confidence level was set at 90\%. In all the scenarios, the respondents had three options to choose; "yes", "no", and "not sure". The "no" and "not sure" responses were put together in one group while "yes" was on the other group. That being the case, the outcome is binary-either "yes" or "no and not sure," which are commonly modeled by either logit or probit models. Usually, logit regression, which assumes the underlying logistic distribution, is preferred due to easy interpretation of the odds ratio [11] [12] [13]. The logistic regression is given by

$$
\operatorname{logit}\left(P_{i}\right)=\ln \left(\frac{P_{i}}{1-P_{i}}\right)=\beta X+\varepsilon
$$

whereby $P_{i}$ be the probability that the respondent chooses "yes" in the presented scenarios, $\beta$ a matrix of the parameters, $X$ a matrix of explanatory variables, and $\varepsilon$ a matrix of the error term. The model interpretation is based on the odds ratio, which is the odds that the respondent will choose "yes" given he/she is a regular user, compared to the odds that he/she will choose "yes" given he/she is the occasional user. The modeling results presented here covers the impact of the DMSs on drivers' decision to detour and the satisfaction of the DMSs message clarity for the given set of parameters.

\subsubsection{Text Analysis}

The text analysis was applied to the unstructured responses, whereby respondents were given a space to write their views. A question such as "Please provide any suggestions to make the dynamic message signs more useful to you. More than one answer is ok.-Other, specify" requires a text analysis approach since it results in unstructured data. In this case, the text network [14], which maps the text from sentence to the network, was used. The network is represented by the nodes and arcs; the larger the node, the higher the frequency that the keyword. Similarly, the thicker the arc between two keywords, the higher the frequency that the two keywords were in the same sentence.

\section{Incidents and DMSs Log Data Analysis and Discussion}

The research team sought to verify whether dynamic message signs have been used in a timely manner to provide incident/congestion information to the mo- 
torists for route diversion on freeways. Specifically, the focus was on the timing, the detail of message content, and the extent of message dissipation. The two weeks (12/2/2014-12/16/2014) of traffic incidents and DMS log data were collected for the freeways that are covered by the DMSs (Figure 2). Fifty-nine incidents occurred during this period. The characteristics of the incidents and the information dissipation are presented and discussed below.

\subsection{Incidents' Characteristics}

In characterizing the incidents, the incident type, number and type of the blocked lane, incident severity, and clearance were the focus due to their importance on the road users' decision to take an alternative route. It can be observed that almost all the incidents (94.9\%) were crashes, while vehicle fire and disabled vehicles incidents were also observed. Furthermore, $80 \%$ of crashes occurred in the traveling while the rest occurred on the shoulder and the ramp. Among the lanes, the right lanes with $35.6 \%$ had the highest percentage of crashes, followed by left lanes and center lanes. The impacts of the crashes on lane blockage show that most crashes (59.3\%) resulted in one lane blockage. The FAST has its specific way of categorizing crashes whereby the incidents are categorized into five categories shown in Table 1. More than half (52.5\%) of those categories of incident severities were categorized as noticeable, while $6.8 \%$ and $5.1 \%$ were significant and severe, respectively. Moreover, the incident characteristics reveal that only about one-third of the crashes (30.5\%) involved towing, which implies that the rest (69.5\%) were self-moved. The quick clear approach, which allows the vehicle involved in the crash to be moved to the shoulder for further investigation given that no fatal crash was involved, was applied for about $10.2 \%$ of the crashes. Three of the incidents were secondary crashes, which resulted from the primary crash within the defined area and time. Only $10.2 \%$ of the crashes occurred during night time.

\subsection{DMSs' Message Dissipation Extent, Timing, and Contents}

In this context, the extent of message dissipation was assessed based on the number of the DMSs displayed the message related to the incident and the estimated distance in miles from the crash location to the activated DMSs. The analysis of the incidents' information posted on the DMSs during the two weeks of data collection revealed that when an incident occurs, the information is mostly posted on one (54\%) or two (36\%) DMSs upstream of the incident location. In rare cases, the 3rd (12\%) and 4th (3\%) DMSs upstream of the incident location are activated. According to Table 2, among 59 crashes, about $86.4 \%$ of the crashes had the standard DMSs upstream where the information could be posted; however, only 32 incidents were posted on the immediate DMSs.

Table 2 also portrays some similarities and differences in information dissipation for the immediate and second sets of the DMSs. Two common observations 
noticed are; first, for both sets of DMSs, the expected delay was not communicated to the road users, and second, there was a similar trend for the message posting timing. There are several observed differences. For the immediate sets of the DMSs, in most of the cases (54.2\%), the crashes were declared/communicated to the road users. Contrarily, only $34.5 \%$ of the time, crashes were declared on the second set of DMSs upstream. On the other hand, the incident location was

Table 1. Incidents characteristics.

\begin{tabular}{|c|c|c|c|}
\hline Variable & Category & Number of incidents & Percentage \\
\hline \multirow{4}{*}{ Incident type } & Crash & 56 & $94.9 \%$ \\
\hline & Vehicle fire & 1 & $1.7 \%$ \\
\hline & Disabled vehicle & 1 & $1.7 \%$ \\
\hline & Special event & 1 & $1.7 \%$ \\
\hline \multirow{7}{*}{ Type of lane blocked } & All lanes & 1 & $1.7 \%$ \\
\hline & Express lanes & 2 & $3.4 \%$ \\
\hline & Center lane(s) & 6 & $10.2 \%$ \\
\hline & Left lane(s) & 17 & $28.8 \%$ \\
\hline & Right lane(s) & 21 & $35.6 \%$ \\
\hline & Shoulder & 10 & $16.9 \%$ \\
\hline & Ramp & 2 & $3.4 \%$ \\
\hline \multirow{4}{*}{ Number of lanes blocked } & None & 10 & $16.9 \%$ \\
\hline & One lane & 35 & $59.3 \%$ \\
\hline & Two lanes & 13 & $22.0 \%$ \\
\hline & Three lanes & 3 & $5.1 \%$ \\
\hline \multirow{5}{*}{ Incident severity } & Negligible & 14 & $23.7 \%$ \\
\hline & Noticeable & 31 & $52.5 \%$ \\
\hline & Significant & 4 & $6.8 \%$ \\
\hline & Severe & 3 & $5.1 \%$ \\
\hline & $\mathrm{N} / \mathrm{A}$ & 7 & $11.9 \%$ \\
\hline \multirow{2}{*}{ Towing involved } & Yes & 18 & $30.5 \%$ \\
\hline & No & 41 & $69.5 \%$ \\
\hline \multirow{2}{*}{ Quick clear } & Yes & 6 & $10.2 \%$ \\
\hline & No & 53 & $89.8 \%$ \\
\hline \multirow{2}{*}{ Ambulance involved } & Yes & 15 & $25.4 \%$ \\
\hline & No & 44 & $74.6 \%$ \\
\hline \multirow{2}{*}{ Secondary crash } & Yes & 3 & $5.1 \%$ \\
\hline & No & 56 & $94.9 \%$ \\
\hline \multirow{2}{*}{ Time of day } & Day time & 53 & $89.8 \%$ \\
\hline & Night time & 6 & $10.2 \%$ \\
\hline
\end{tabular}


Table 2. Summarized dissipated information on the DMSs.

\begin{tabular}{|c|c|c|c|c|c|}
\hline \multirow[b]{2}{*}{ Variable } & \multirow[b]{2}{*}{ Category } & \multicolumn{2}{|c|}{ An immediate set of DMSs } & \multicolumn{2}{|c|}{ The second set of DMSs } \\
\hline & & $\begin{array}{l}\text { Number of } \\
\text { crashes }\end{array}$ & Percentage & $\begin{array}{c}\text { Number of } \\
\text { crashes }\end{array}$ & Percentage \\
\hline \multirow{2}{*}{ DMS type } & Standard & 51 & $86.4 \%$ & 48 & $82.8 \%$ \\
\hline & Travel Time & 8 & $13.6 \%$ & 10 & $17.2 \%$ \\
\hline \multirow{2}{*}{$\begin{array}{l}\text { Incident info } \\
\text { posted }\end{array}$} & Yes & 32 & $54.2 \%$ & 21 & $36.2 \%$ \\
\hline & No & 27 & $45.8 \%$ & 37 & $63.8 \%$ \\
\hline \multirow{2}{*}{$\begin{array}{l}\text { Incident type } \\
\text { posted }\end{array}$} & Yes & 32 & $54.2 \%$ & 20 & $34.5 \%$ \\
\hline & No & 27 & $45.8 \%$ & 38 & $65.5 \%$ \\
\hline \multirow{2}{*}{$\begin{array}{l}\text { Incident } \\
\text { location }\end{array}$} & Yes & 29 & $49.2 \%$ & 21 & $36.2 \%$ \\
\hline & No & 31 & $52.5 \%$ & 37 & $63.8 \%$ \\
\hline \multirow{2}{*}{$\begin{array}{l}\text { Severity } \\
\text { blockage }\end{array}$} & Yes & 27 & $45.8 \%$ & 19 & $32.8 \%$ \\
\hline & No & 32 & $54.2 \%$ & 39 & $67.2 \%$ \\
\hline \multirow{2}{*}{ Severity delay } & Yes & 2 & $3.4 \%$ & 1 & $1.7 \%$ \\
\hline & No & 57 & $96.6 \%$ & 57 & $98.3 \%$ \\
\hline \multirow{4}{*}{$\begin{array}{l}\text { Message posting } \\
\text { timing (min) }\end{array}$} & 0 & 11 & $18.6 \%$ & 8 & $13.8 \%$ \\
\hline & 1 to 5 & 15 & $25.4 \%$ & 10 & $17.2 \%$ \\
\hline & 5 to 10 & 4 & $6.8 \%$ & 2 & $3.4 \%$ \\
\hline & $>10$ & 1 & $1.7 \%$ & 0 & $0.0 \%$ \\
\hline \multirow{6}{*}{$\begin{array}{l}\text { Message } \\
\text { dissipated } \\
\text { distance } \\
\text { upstream the } \\
\text { crash (mi) }\end{array}$} & $0-0.5$ & 6 & $10.2 \%$ & 0 & $0.0 \%$ \\
\hline & $0.5-1.0$ & 7 & $11.9 \%$ & 0 & $0.0 \%$ \\
\hline & $1.0-1.5$ & 4 & $6.8 \%$ & 1 & $1.7 \%$ \\
\hline & $1.5-2.0$ & 6 & $10.2 \%$ & 2 & $3.4 \%$ \\
\hline & $2.0-3$ & 6 & $10.2 \%$ & 4 & $6.9 \%$ \\
\hline & $>3.0$ & 2 & $3.4 \%$ & 14 & $24.1 \%$ \\
\hline \multirow{2}{*}{$\begin{array}{c}\text { Exit Ramp } \\
\text { present }\end{array}$} & Yes & 32 & $54.2 \%$ & 57 & $98.3 \%$ \\
\hline & No & 27 & $45.8 \%$ & 1 & $1.7 \%$ \\
\hline
\end{tabular}

communicated by $49.2 \%$ and $36.2 \%$ on the first and the second sets of the DMSs, respectively, while lane blockage information was posted for 27 incidents (45.8\%) and 19 incidents (32.8\% on the first and second sets of DMSs respectively. Even though much of the useful information were communicated on the immediate DMSs, the distance from those DMSs to the crash location is short, and there might be no exit ramp where the drivers can detour. Table 2 shows that $54.2 \%$ of the incidents had the exit ramps between the crash location and the immediate set of DMSs which is very contrary to the second set of the DMSs with $98.3 \%$ of the exit ramp's presence between the crash location and DMSs.

Through these observations, it can be concluded that most of the time, travelers are informed of the incident type (crash, disabled vehicle, vehicle fire etc.), incident location, and blockage, while the delay expected is not too common to 
be posted. However, most of this information is posted on the immediate DMSs, which implies that even though travelers may have been informed of the crash downstream, they are less likely to take detours due to the exit ramps' absence. The next segment presents the survey questionnaire results, which depict the roadway users' perceptions of the preference and usefulness of the DMSs.

\section{Survey Questionnaire Analysis and Discussion}

To evaluate whether the Dynamic Message Signs in the Las Vegas area present the incident/congestion information in the right way, the Qualtrics based surveys were designed and distributed to the travelers and truckers through the web links. In the survey, respondents were asked about where they live in the Las Vegas area, how frequently they use the freeway systems, their attitude to using an alternative route. Furthermore, whether the DMSs help them take alternative routes, whether the DMS presents sufficient information about crashes critical to their choice of routes, and whether the DMS presents the information in the right form. A total of 238 responses were collected after two months of survey distribution. Demographically, the respondents' ages were normally distributed where the young and senior travelers were fewer than those middle-aged. This distribution represents a typical driving population, with about $40 \%$ of the responses being male and $60 \%$ being female. This proportion is close to the reality perceived. Education-wise, about $21 \%$ of the respondents have other degrees, and $31 \%$ do not disclose their education.

\subsection{Preferred Method for Incident Information Dissipation}

Apart from DMSs, there are other means of information dissipation on and off the freeway tested and implemented in other locations. On the freeway, two competing means; Travel time signs (Figure 3(b)), dynamic display board Figure 3(c)) have been used [7] while Phone app, radio, social media, commercial traveler info, public traveler info are used for both off and on the freeway [15] [16]. The dynamic traffic display board, which is popular in other countries, is presented to travelers to seek their opinion. Drivers were asked to compare this board with the existing DMS and travel time signs by ranking them. Further, drivers were asked whether they prefer other information dissipation means apart from the three mentioned above.

The results in Figure 4(a) indicate that about $70 \%$ of the time, DMS was chosen as the best. The responses' distribution shows that the survey respondent had a similar preference for time signs and map board, as shown by the percentage of distribution of the ranks (Figure 4(a)). Both travel time signs and map boards are ranked about $15 \%, 40 \%$, and $45 \%$, as the first, second, and third, respectively. Most respondents ranked the DMS as their first choice, ranked travel time, and map board as the second and third options. Further, the distribution of the rankings was evaluated per the driver's decision to detour with respect to the message displayed on the DMSs. Drivers who do not detour until they get the message from the DMSs were composed of the largest percent of the res- 
pondents, while those who are not sure constitutes the smallest percentage. Regardless of the decision to detour, there was a consistent pattern in ranking the three travel information options on the freeways.

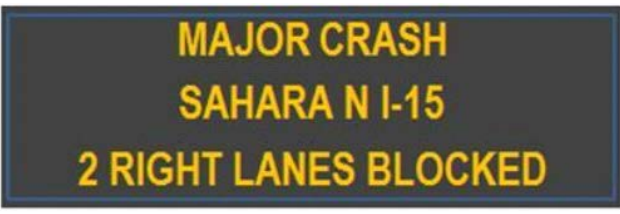

(a) Dynamic Message Sign

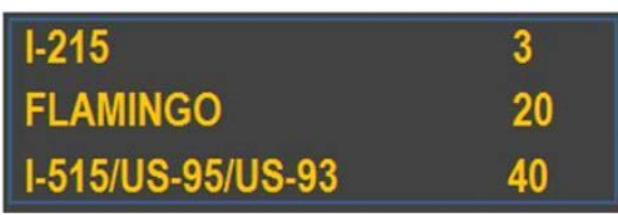

(b) Traved Time Sign

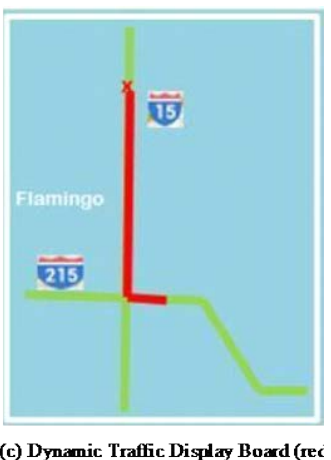

shows congested area)

Figure 3. Alternative options for incident information dissipation.

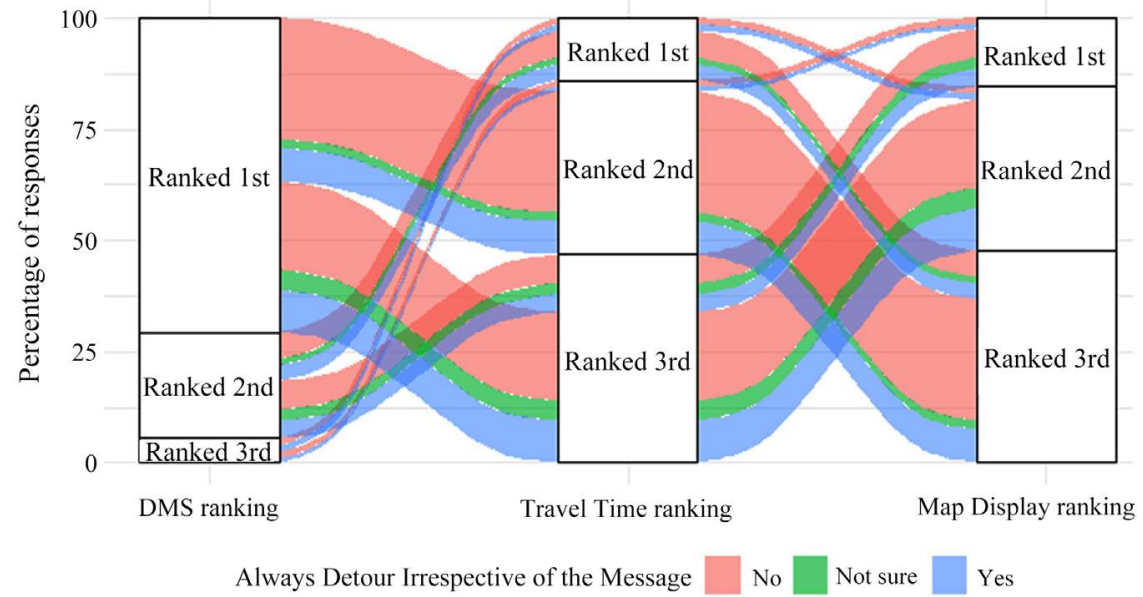

(a)

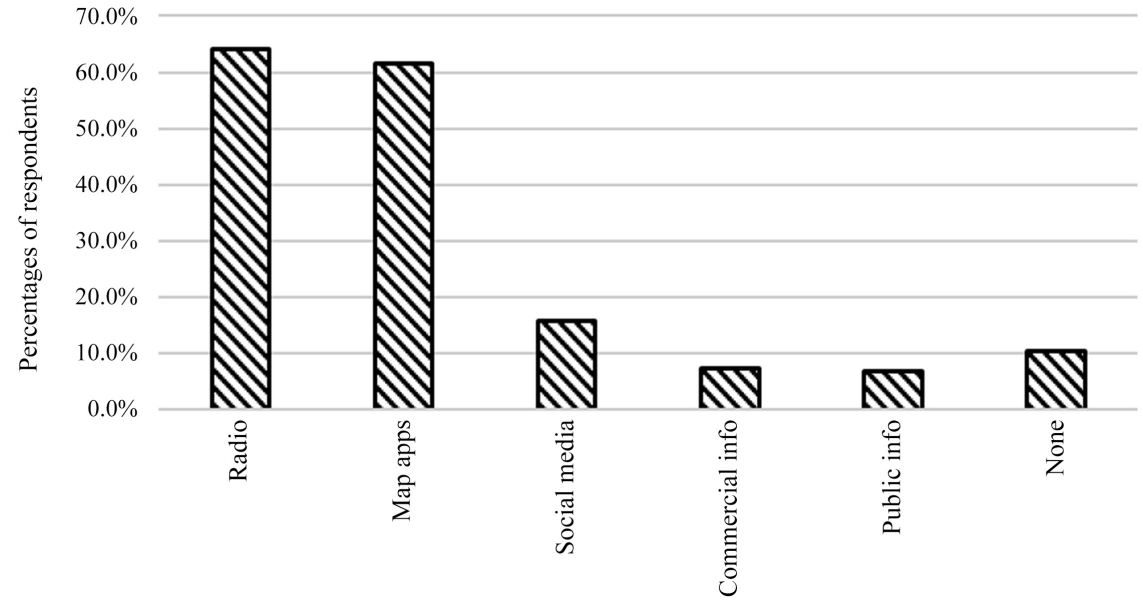

(b)

Figure 4. Preferred methods for incident information dissipation. (a) DMSs preference over other static methods; (b) Other preferred methods. 
Additionally, in their response regarding other travel information sources, radio and mobile apps outscored other means as they had $64 \%$ and $62 \%$ respectively, while social media (Twitter) scored relatively small percent (15.8\%). Public traveler information services (e.g., RTC traffic map, 511) and Commercial traveler information services (e.g., INRIX, TomTom) had a very small percentage, as shown in Figure 4(b).

\subsection{Freeway Users' DMSs Dependency on Detouring Decision}

In this section, a comparison between two groups of drivers, regular users and occasional users of the freeways were compared. The authors intended to explore whether the familiarity with the freeways has an impact on the decision that could affect the effectiveness of the DMSs. The regular freeway users (daily users) comprised $81 \%$ of all the respondents, while occasional users (those who use freeway once per week or less) comprised the remaining $29 \%$.

The logistic regression results in Table 3 revealed that, provided that no information is dissipated, regular freeway users are $25 \%$ less likely to take an alternative route than the occasional users (Table 3(a)).

Furthermore, the regular freeway users are DMSs dependents, as shown by the DMSs message results. As per Table 3(b), regular freeway users are less likely to take an alternative route after seeing the congestion regardless of the DMSs messages. This is to say; regular users are more DMSs dependent than occasional users. However, the p-value of 0.677 revealed no statistically significant difference between regular and occasional users on taking alternative routes regardless of the DMSs messages. Regarding taking the alternative routes, given the accurate information on the DMSs, the logistic results in Table 3 (c) revealed that there is a statistically significant ( $\mathrm{p}$-value $=0.026$ ) difference between regular and occasional freeway users. In fact, regular freeway users are almost three times (Odd ratio $=2.954$ ) more likely to take alternative routes when accurate information is provided.

\subsection{Freeway Users' Satisfaction on DMSs Messages Contents}

To improve the message contents, the authors wanted to know how the existing contents satisfy the road users. The respondents were posed with the question regarding their general satisfaction with the DMSs information and the specific details such as incident location, fatality, time, and backup. The analysis revealed that about $63 \%$ of the daily freeway users and $16 \%$ of the occasional users agree that the information posted on DMSs are useful, while $7.5 \%$ and $1.4 \%$ of the daily and occasional freeway users were not sure. The logistic regression results (Table 4(a)) revealed that regular freeway users perceived that the information posted on the DMSs is not useful in general. However, with a p-value of 0.491 , this difference is not statistically significant at the $5 \%$ level. Therefore, both regular and occasional users perceive that the information posted on the DMSs is less useful. On the specific DMSs posted messages, there was observed variation 
on the level of satisfaction for each detail by the level of freeway usage. However, only clarity of the information related to the incident location (Table 4(b)) was statistically significantly different at the $5 \%$ level ( $\mathrm{p}$-value $=0.063$ ) for regular and casual freeway users. Being specific, regular freeway users are two times more likely to be satisfied with the details posted on the DMSs regarding the incident location. Although the remained DMSs message contents revealed a non-statistically significant at $5 \%$ level, some valuable information can be acquired. For instance, the coefficients for all the message contents details (Tables 4(c)-(d)) are positive, which implies that road users are satisfied with the information posted on DMSs regarding the fatality, blockage, and time. On the other hand, the incident backup details' negative coefficient shows that the freeway users are less satisfied with the information posted on the DMSs regarding the backup.

Table 3. Logistic Regression Results for Driver's Dependency on DMSs For Detouring Decision. (a) Take alternative route when run into congestion; (b) Take alternative route after seeing congestion regardless DMS message; (c) Take alternative route if accurate information is provided on DMSs.

(a)

\begin{tabular}{cccccc}
\hline Freeway usage & Coefficient & Odds Ratio & Std. Error & Z-statistic & P-value \\
\hline Regular user & -1.386 & 0.250 & 0.624 & -2.220 & 0.026 \\
constant & 2.590 & 13.333 & 0.599 & 4.330 & 0.000 \\
& & Number of observations $=225$, LR chi2 $(1)=6.79$ \\
Model parameters & Prob > chi2 $=0.0092$, Pseudo R2 $=0.0301$ \\
& & Log likelihood $=-109.19774$ \\
\hline
\end{tabular}

(b)

\begin{tabular}{cccccc}
\hline Freeway usage & Coefficient & Odds Ratio & Std. Error & Z-statistic & P-value \\
\hline Regular user & -0.155 & 0.857 & 0.372 & -0.420 & 0.677 \\
constant & -0.836 & 0.433 & 0.332 & -2.520 & 0.012 \\
& & Number of observations $=224$, LR chi2 $(1)=0.17$ & \\
Model parameters & & Prob $>$ chi2 $=0.6792$, Pseudo R2 $=0.0006$ \\
& & Log likelihood $=-132.05026$ \\
\end{tabular}

(c)

\begin{tabular}{cccccc}
\hline Freeway usage & Coefficient & Odds Ratio & Std. Error & Z-statistic & P-value \\
\hline Regular user & 1.083 & 2.954 & 0.486 & 2.230 & 0.026 \\
constant & 1.476 & 4.375 & 0.392 & 3.770 & 0.000
\end{tabular}

Number of observations $=224, \operatorname{LR} \operatorname{chi} 2(1)=4.55$

Model parameters $\quad$ Prob $>$ chi $2=0.0329$, Pseudo $R 2=0.0327$

Log likelihood $=-67.416563$ 
Table 4. DMSs General and Specific Usefulness. (a) General usefulness of DMSs posted information; (b) DMS messages clarity about incident location; (c) DMS messages clarity about incident fatality; (d) DMS messages clarity about incident blockage; (e) DMS messages clarity about incident elapsed time; (f) DMS messages clarity about incident backup.

(a)

\begin{tabular}{cccccc}
\hline Freeway usage & Coefficient & Odds Ratio & Std. Error & Z-statistic & P-value \\
\hline Regular user & -0.313 & 0.731 & 0.454 & -0.690 & 0.491 \\
constant & 1.580 & 4.857 & 0.415 & 3.810 & 0.000 \\
& \multicolumn{5}{c}{ Number of observations = 214, LR chi2 $(1)=0.5$} \\
Model parameters & Prob $>$ chi2 $=0.481$, Pseudo R2 $=0.0023$ \\
\multicolumn{5}{c}{ Log likelihood $=-109.818$} \\
\hline
\end{tabular}

(b)

\begin{tabular}{cccccc}
\hline Freeway usage & Coefficient & Odds Ratio & Std. Error & Z-statistic & P-value \\
\hline Regular user & 0.741 & 2.098 & 0.399 & 1.860 & 0.063 \\
constant & 0.882 & 2.417 & 0.343 & 2.570 & 0.010 \\
& \multicolumn{5}{c}{ Number of observations $=217$, LR chi2 $(1)=2.26$} \\
Model parameters & Prob $>$ chi2 $=0.071$, Pseudo R2 $=0.0155$ \\
\multicolumn{5}{c}{ Log likelihood $=-103.546$} \\
\hline
\end{tabular}

(c)

\begin{tabular}{cccccc}
\hline Freeway usage & Coefficient & Odds Ratio & Std. Error & Z-statistic & P-value \\
\hline Regular user & 0.063 & 1.065 & 0.359 & 0.180 & 0.860 \\
constant & -0.550 & 0.577 & 0.324 & -1.700 & 0.090 \\
& & Number of observations $=217$, LR chi2 $(1)=0.03$ \\
Model parameters & Prob > chi2 $=0.8598$, Pseudo R2 $=0.0001$ \\
& \multicolumn{4}{c}{ Log likelihood $=-143.859$} \\
\end{tabular}

(d)

\begin{tabular}{|c|c|c|c|c|c|}
\hline Freeway usage & Coefficient & Odds Ratio & Std. Error & Z-statistic & P-value \\
\hline Regular user & 0.190 & 1.210 & 0.353 & 0.540 & 0.590 \\
\hline \multirow[t]{2}{*}{ constant } & 0.345 & 1.412 & 0.317 & 1.090 & 0.277 \\
\hline & \multicolumn{5}{|c|}{ Number of observations $=217, \operatorname{LR}$ chi2 $(1)=0.29$} \\
\hline \multirow[t]{2}{*}{ Model parameters } & \multicolumn{5}{|c|}{ Prob $>$ chi $2=0.5916$, Pseudo $R 2=0.0001$} \\
\hline & \multicolumn{5}{|c|}{ Log likelihood $=-143.731$} \\
\hline
\end{tabular}

(e)

\begin{tabular}{cccccc}
\hline Freeway usage & Coefficient & Odds Ratio & Std. Error & Z-statistic & P-value \\
\hline $\begin{array}{c}\text { Regular user } \\
\text { constant }\end{array}$ & 0.372 & 1.451 & 0.412 & 0.900 & 0.367 \\
& -1.269 & 0.281 & 0.377 & -3.360 & 0.001
\end{tabular}

Number of observations $=217, \operatorname{LR} \operatorname{chi} 2(1)=0.85$

Model parameters $\quad$ Prob $>$ chi2 $=0.3561$, Pseudo R2 $=0.0033$

Log likelihood $=-127.521$ 
(f)

\begin{tabular}{cccccc}
\hline Freeway usage & Coefficient & Odds Ratio & Std. Error & Z-statistic & P-value \\
\hline Regular user & -0.015 & 0.986 & 0.347 & -0.040 & 0.967 \\
constant & 0.049 & 1.050 & 0.312 & 0.160 & 0.876 \\
& \multicolumn{4}{c}{ Number of observations $=216$, LR chi2 $(1)=0$} \\
Model parameters & Prob > chi2 $=0.9667$, Pseudo R2 $=0$ \\
& Log likelihood $=-149.681$ \\
\hline
\end{tabular}

\section{Suggested Improvements}

The analysis in the previous parts of this study has shown that in most cases, there is no statistically significant difference between different levels of freeway users towards various posed scenarios. This part, therefore, presents the analysis that combines both regular and occasional freeway users. The respondents were also asked to suggest the improvements so that the DMSs may be more useful and effective. In this question, respondents could select multiple pre-set options and/or provide their suggestions.

Respondents were asked to choose among the following suggested improvements; provision of information earlier, provision of congestion length information, provision of congestion duration, adding more DMSs or write their own suggestion in the "other" option. The analyzed responses (Figure 5(a)) show that most travelers (73\%) suggest that the congestion information should be given as early as possible. This can be done by posting this information on more than one DMS upstream of the incident location. The suggested improvements for congestion duration and length information were ranked second, with roughly $54 \%$ of responses. The need for more DMS signs on the freeway was preferred by about $50 \%$ of the respondents, while $20 \%$ selected the "other" category and provided their detailed needs.

Regardless of having a small percentage of respondents, the "other" category might be more realistic and helpful than most pre-set selections since respondents provided their vision. Since the comments provided in the "other" category were in the form of unformatted text, the authors used text analysis approaches to explore the comments' content. The summary of the results is presented in Figure 5(b)). It can be observed that in the "other" category, most of the respondents suggested the provision of an alternative route as revealed by the two keywords "alternative" and "route" and a thick link between them. Accident clearing information, the use of dynamic board to display the affected exits so that the road users may plan accordingly, and such information should be flashing by using the traditional DMS. Furthermore, provision of accurate and updated information on the occurrence and expected clearance time was also listed. Other minor but important comments included cooperating with other agencies with a high number of freeway users such as government offices, universities, hotels, etc., and companies dealing with traffic incidents such as Tom tom. 


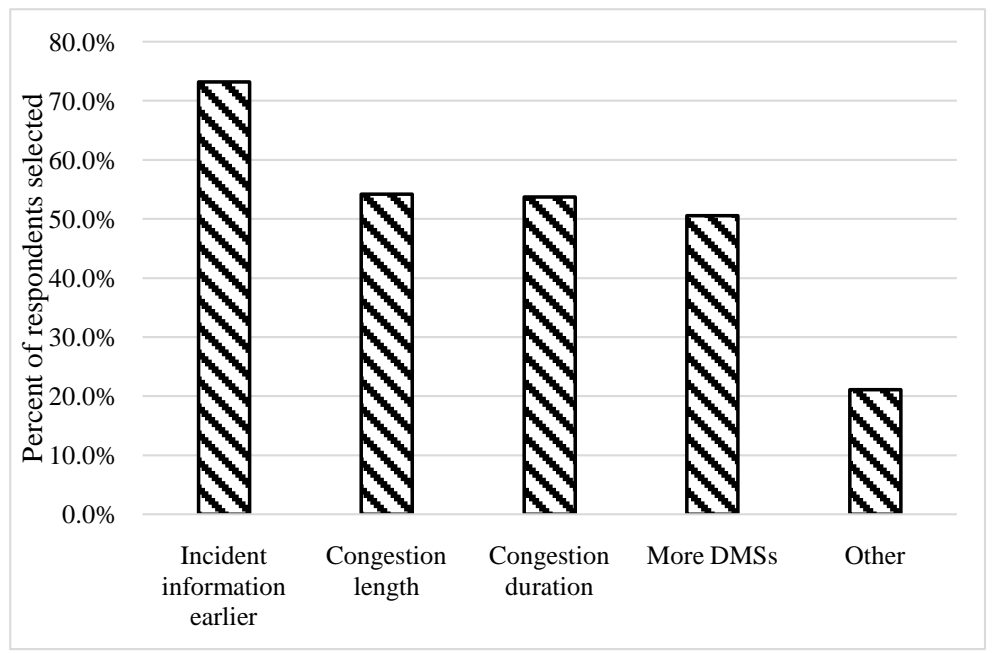

(a)

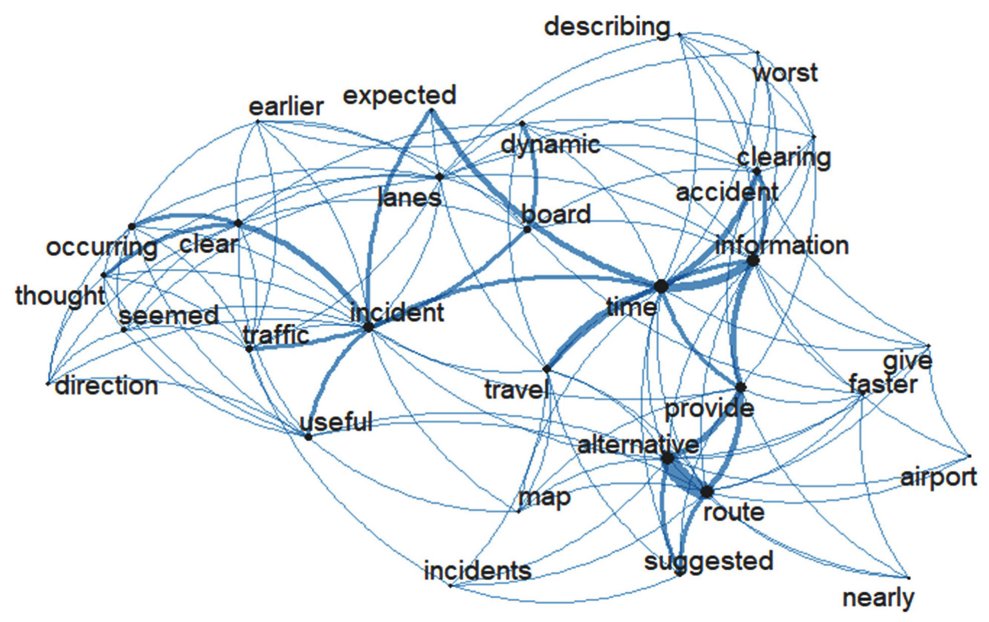

(b)

Figure 5. Suggestions to improve dynamic message Signs. (a) Selected suggested improvements; (b) Other specified improvements.

\section{Conclusion and Recommendations}

This study evaluated the usefulness of the DMSs on the freeways in Las Vegas, Nevada. Two weeks of incident data, DMSs' log data, and the survey questionnaire were used for evaluation. The incident data was used to determine the type and severity of the incident. The DMSs logs were used to determine the message's content dissipated, the extent of dissipation, and the timing. The survey questionnaire on the other hand was used to determine the impact of the DMSs messages on detour decision, drivers' satisfaction with the details provided on the DMSs, preference of DMS against the other means of information dissipation on and off the roadway, and the suggested improvements.

The analysis of the incident and DMS log data revealed that most of the time (54.2\%), travelers get informed about the presence of the incident (crash, disabled vehicle, vehicle fire etc.). Regarding the message content, roadway users were informed of the type of the incident, location, and the lane blockage, while 
the expected delays or the time the incident occurred were infrequently posted. The incident information is mostly posted on the immediate DMSs (54\%) or two (36\%) successive DMSs upstream the incident location. The survey questionnaire results revealed that standard DMSs are the most preferred sources of traffic information on the freeway compared to the travel time only DMSs, and the graphical map boards. The perceived DMSs' impact on the detour decision was different for regular and occasional freeway users when the accurate information is posted on the DMSs. Further, regular freeway users perceive that the incident location information was clearly disseminated to the road users. Conversely, both regular and occasional freeway users showed no difference regarding the incident fatality, blockage, backup, and duration information. The suggested improvements included information to be provided earlier and improvements on congestion duration and length information.

In conclusion, considering the DMSs log and the survey responses results, it can be concluded that 1) both DMSs log data and survey responses show that to some extent, the incident information is dissipated to drivers through DMSs; 2) there is some information that drivers perceive they are important but are not posted on the DMSs; 3 ) there is slight difference in perception of what is being offered on the DMSs between the regular and occasional freeway users, thus, the improvements on the DMSs will benefit both types of freeway users. The DMSs operators can use the findings from this study to improve travel time dissemination in Nevada and other states where the DMSs are being utilized as the means of incident information dissipation on the freeways. The operators can understand the deficiency of what they provide to what the road users want. Provision of road users suggested improvements would increase the detour rates.

\section{Acknowledgements}

The research team would like to thank Mr. Brian Hoeft and his staff at FAST who has been providing the assistance needed for this project.

\section{Conflicts of Interest}

The authors declare no conflicts of interest regarding the publication of this paper.

\section{References}

[1] Dudek, C.L. (2004) Changeable Message Sign Operation and Messaging Handbook. Washington DC.

https://tmcpfs.ops.fhwa.dot.gov/cfprojects/uploaded_files/CMS\%20Operation\%20a nd\%20Messaging\%20Handbook-Final\%20Draft.pdf

[2] Dudek, C.L. (2006) Dynamic Message Sign Message Design and Display Manual. Washington DC.

https://static.tti.tamu.edu/tti.tamu.edu/documents/0-4023-P3.pdf

[3] Huo, H. and Levinson, D. (2006) Effectiveness of VMS Using Empirical Loop Detector Data.

http://www.path.berkeley.edu/sites/default/files/publications/PWP-2006-04.pdf 
[4] Edara, P., Sun, C., Keller, C. and Hou, Y. (2014) Evaluation of Dynamic Message Signs on Rural Freeways: Case Study of a Full Freeway Closure. Journal of Transportation Engineering, 140, 89-98. https://doi.org/10.1061/(ASCE)TE.1943-5436.0000614

[5] Haghani, A., et al. (2013) Evaluation of Dynamic Message Signs and Their Potential Impact on Traffic Flow. Report, MD-13-SP109B4C, Maryland State Highway Administration.

[6] Guattari, C., De Blasiis, M.R. and Calvi, A. (2012) The Effectiveness of Variable Message Signs Information: A Driving Simulation Study. Procedia-Social and Behavioral Sciences, 53, 692-702. https://doi.org/10.1016/j.sbspro.2012.09.919

[7] Yan, X. and Wu, J. (2014) Effectiveness of Variable Message Signs on Driving Behavior Based on a Driving Simulation Experiment. Discrete Dynamics in Nature and Society, 2014, Article ID: 206805. https://doi.org/10.1155/2014/206805

[8] Demetsky, M.J. and Schroeder, J.L. (2010) Evaluation of Driver Reactions for Effective Use of Dynamic Message Signs in Richmond, Virginia.

https://rosap.ntl.bts.gov/view/dot/20229

[9] FAST (2016) Dynamic Message Signs. http://bugatti.nvfast.org/PMMS/DMSs.aspx

[10] Teng, H., Kutela, B. and Hu, B. (2015) Field Test of Slow Moving Traffic Alerting System on Freeways in Las Vegas, Nevada Assessment of the Effectiveness of the Dynamic Message Signs on the Freeways in Las Vegas, Nevada. Nevada Department of Transportation. https://rosap.ntl.bts.gov/view/dot/35044

[11] Woodridge, J.M. (2012) Introductory Economics A Modern Approach. 5th Edition, South-Western, Cengage Learning, Mason.

[12] Kutela, B. and "Harry" Teng, H. (2020) Assessment of Methodological Alternatives for Modeling the Spatiotemporal Crossing Compliance of Pedestrians at Signalized Midblock Crosswalks. Journal of Transportation Engineering, Part A: Systems, 146, Article ID: 04019062. https://doi.org/10.1061/JTEPBS.0000300

[13] Kutela, B. and Teng, H. (2020) Evaluating the Influential Factors for Pushbutton Utilization at Signalized Midblock Crosswalks. Safety Science, 122, Article ID: 104533. https://doi.org/10.1016/j.ssci.2019.104533

[14] Benoit, K., et al. (2018) Quanteda: An R Package for the Quantitative Analysis of Textual Data. Journal of Open Source Software, 3, 774. https://doi.org/10.21105/joss.00774

[15] Wang, D., Xiang, Z. and Fesenmaier, D.R. (2016) Smartphone Use in Everyday Life and Travel. Journal of Travel Research, 55, 52-63. https://doi.org/10.1177/0047287514535847

[16] Wang, D. and Fesenmaier, D.R. (2013) Transforming the Travel Experience: The Use of Smartphones for Travel. In: Information and Communication Technologies in Tourism 2013, Springer, Berlin, 58-69.

https://doi.org/10.1007/978-3-642-36309-2_6 